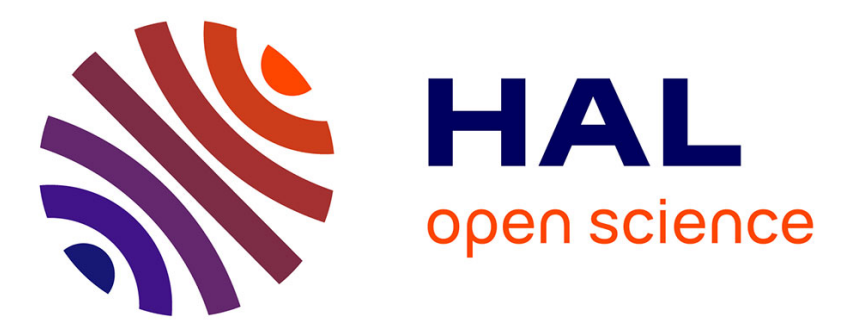

\title{
Interconnected delay and state observer for nonlinear systems with time-varying input delay
}

Vincent Léchappé, Emmanuel Moulay, Franck Plestan, Alain Glumineau

\section{To cite this version:}

Vincent Léchappé, Emmanuel Moulay, Franck Plestan, Alain Glumineau. Interconnected delay and state observer for nonlinear systems with time-varying input delay. 2016 American Control Conference (ACC 2016), Jul 2016, Boston, MA, United States. 10.1109/ACC.2016.7524894 hal-01350876

\section{HAL Id: hal-01350876 https://hal.science/hal-01350876}

Submitted on 2 Aug 2016

HAL is a multi-disciplinary open access archive for the deposit and dissemination of scientific research documents, whether they are published or not. The documents may come from teaching and research institutions in France or abroad, or from public or private research centers.
L'archive ouverte pluridisciplinaire $\mathbf{H A L}$, est destinée au dépôt et à la diffusion de documents scientifiques de niveau recherche, publiés ou non, émanant des établissements d'enseignement et de recherche français ou étrangers, des laboratoires publics ou privés. 


\title{
Interconnected delay and state observer for nonlinear systems with time-varying input delay
}

\author{
V. Léchappé, E. Moulay, F. Plestan and A. Glumineau
}

\begin{abstract}
This work presents a general framework to estimate both state and delay thanks to two interconnected observers. This scheme can be applied to a large class of nonlinear systems with time-varying input delay. In order to illustrate this approach, a new delay observer based on an optimization technique is proposed. Theoretical results are illustrated and compared with existing works in simulation.
\end{abstract}

Index Terms - Input delay, interconnected observers, timedelay estimation, state observation.

\section{INTRODUCTION}

State observation is very useful to design output feedback when the whole state is not available. Many techniques have been developed such as Luenberger observer for linear systems [17], Kalman filter for linear time varying systems [10], high-gain observer for uniformly observable systems [8]. These observers have been designed for delay free systems. For delay systems, standard state observers can often be adapted when the delay is known [9], [11], [24]. Although some works deal with state observation with unknown delay [19], [21], very few articles tackle the issue of both state and delay estimation.

In practical applications, the input delay is often impossible to measure but it is also often very prejudicial for the stability of the system. The standard way to stabilize such systems is to use predictive controllers [1], [13], [15]. However, the delay has to be known to compute the prediction. Therefore, estimating the delay is an essential challenge. First works about delay estimation in the control field often consider the delay as a parameter and apply identification techniques [2], [23]. A larger description of delay estimation techniques is provided in [14].

Very recently, some works have arisen about state observation and delay estimation. In [22], parameter, state and delay estimation is proposed for chaotic and hyperchaotic delayed systems. In [5], the authors present an observation technique for linear systems that may be seen as a specific case of the more general framework treated here. A method based on a particular sample/hold technique is given in [7] but no interconnection is made between delay estimation and state observation. Finally, a new observation method is proposed in [14]. In this latter work, the delay is considered as a part of an extended state and a single observer estimates both state and delay; so it is not possible to tune them separately

Vincent Léchappé, Franck Plestan and Alain Glumineau are with LUNAM Université, Ecole Centrale de Nantes, IRCCyN UMR CNRS 6597, Nantes, France. Firstname.Name@irccyn.ec-nantes.fr. Emmanuel Moulay is with Xlim, UMR CNRS 7252, Université de Poitiers emmanuel.moulay@univ-poitiers.fr because the observer has only one gain that governs the convergence speed of the state and the delay estimation.

The contribution of the present work is to provide a new structure to observe both the delay and the state for SISO nonlinear systems. The first contribution is to use an interconnected scheme to decouple delay observation from state observation and thus to allow a separate tuning of both observers. This is particularly important for input delay systems whose delay dynamics is often very different from system dynamics. The second contribution is the design of an original delay observer, based on the combination of an Extended System Observer (ESO) and an optimization method.

This paper is organized as follows. The problem statement is presented in Section II. Then, a general result for state and delay interconnected observers is provided in Section III. In Section IV, a state observer and a delay observer are shown to satisfy the conditions of the general scheme. The performance of the interconnected observer is confirmed by a simulation analysis in Section V. Finally, some conclusions are drawn in Section VI.

\section{Problem Statement}

In this paper, a class of SISO nonlinear systems with a time-varying and unknown delay $\tau(t)$ in the input $u$ is considered. These systems are defined as follows

$$
\left\{\begin{array}{l}
\dot{x}=\Lambda x+f(x)+g(y) u(t-\tau(t)) \\
y=C x=x_{1} \\
x(0)=x_{0}
\end{array}\right.
$$

with $x=\left[x_{1}, \ldots, x_{n}\right]^{T} \in \mathbb{R}^{n}, y \in \mathbb{R}, u \in \mathbb{R}$,

$$
\begin{gathered}
\wedge=\left[\begin{array}{cc}
0 & I_{n-1} \\
0 & 0
\end{array}\right], \quad g(y)=[0, \ldots, G(y)]^{T}, \\
f(x)=\left[\begin{array}{c}
f_{1}\left(x_{1}\right) \\
f_{2}\left(x_{1}, x_{2}\right) \\
\vdots \\
f_{n}\left(x_{1}, \ldots, x_{n}\right)
\end{array}\right] \quad \text { and } C=[1,0, \ldots, 0] .
\end{gathered}
$$

In the sequel, the following assumptions are used.

Assumption 1: The functions $f$ and $G$ are respectively in $C^{1}\left(\mathbb{R}^{n}\right)$ and $C^{1}(\mathbb{R})$ and $f$ is globally Lipschitz, i.e. there exists $c_{1}>0$ such that for $(x, z) \in \mathbb{R}^{n} \times \mathbb{R}^{n}$

$$
\|f(x)-f(z)\| \leq c_{1}\|x-z\| \text {. }
$$

Assumption 2: The function $G$ is bounded, i.e. there exists $\bar{G}>0$ such that for all $y \in \mathbb{R}$

$$
|G(y)| \leq \bar{G} \text {. }
$$


Assumption 3: The delay is time-varying and unknown and $\tau \in\left[\tau_{\min }, \tau_{\max }\right]$ with $\tau_{\min }, \tau_{\max }>0$. Its dynamics is denoted $\dot{\tau}(t)=\eta_{\tau}(t)$.

The subscript $t$ will sometimes be omitted to make the presentation clearer.

Assumption 4: The input $u$ is twice time differentiable and its time derivatives are bounded, i.e. there exist constant $U_{1}, U_{2}>0$ such that for all $t \geq-\tau_{\max }$

$$
|\dot{u}(t)| \leq U_{1} \quad \text { and } \quad|\ddot{u}(t)| \leq U_{2} .
$$

Thanks to the triangular form of matrix $\Lambda$, it is clear that system (1) is observable with respect to $x$. As for the delay, the observability condition is strongly related to the properties of the input signal $u$. The observability condition is given below

Theorem 1: The delay $\tau$ is observable if and only if

$$
G(y(t)) \neq 0 \quad \forall t \geq 0
$$

and

$$
\dot{u}(t) \neq 0 \quad \forall t \geq-\tau_{\max } .
$$

The proof is similar to the one given in [14]. Condition (3) is quite natural because if the input is constant, the delay has no influence on the system so it cannot be observed. Nevertheless, it is restrictive because it means that the input should be strictly monotonic. Similarly, the condition on $G(y)$ can be restrictive. In practice, the notion of persistence can be used to relax these conditions [3]. Condition (3) justifies the assumption introduced by [6]

Assumption 5: There are time intervals $I$ sufficiently large where the first derivative of $u$ is continuous and bounded: for all $t \in I$,

$$
\alpha<|\dot{u}(t)|
$$

with $\alpha>0$.

Indeed, if (4) holds then (3) is automatically verified on $I$. Conditions (2) and (4) are assumed to be verified in the rest of the paper. A new general scheme is proposed in the next section to estimate both the delay and the state of system (1) thanks to two interconnected observers.

\section{INTERCONNECTED STATE AND DELAY OBSERVERS}

The scheme is summarized on Figure 1. Especially, all state observers existing for system with known delay can be used (Luenberger, Kalman, high-gain, sliding-mode...). In this section, a general framework is considered and some particular observers will be studied in Section IV.

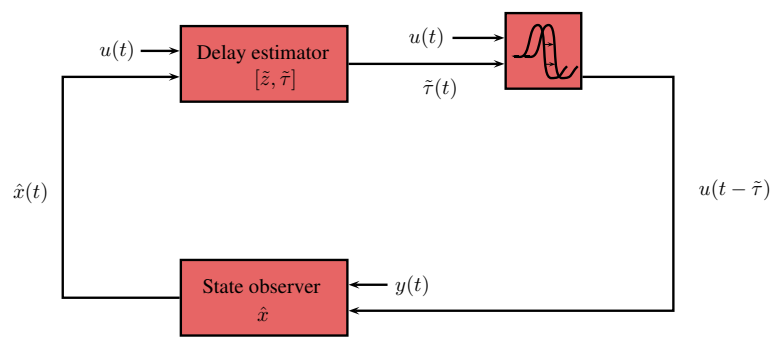

Fig. 1. State observer interconnected with delay observer
Following the notation of Figure 1, the state estimation error is denoted

$$
e_{x}=\hat{x}-x
$$

and the errors of the delay observer are

$$
e_{z}=\tilde{z}-z \quad \text { and } \quad e_{\tau}=\tilde{\tau}-\tau .
$$

The variable $\tilde{z}$ in the delay observer block of Figure 1 is an auxiliary variable that will be useful to compute an estimate of the delay. The $z$ variable can contain for example the input $u(t-\tau)$ and its time derivative as shown in the particular example of Section IV-B. In the sequel, $V_{x}$ (respectively $V_{z h}$ ) will refer to a Lyapunov candidate function for the state observer (respectively the delay observer). These two observers are connected by the estimated delay $\tilde{\tau}$ that enters the state observer through $u(t-\tilde{\tau})$ and by the state estimation $\hat{x}$ that enters the delay observer. To study the convergence of the whole system, the interconnected subsystems approach is going to be used. As it will be useful in the sequel, some notations are reminded:

- A continuous function $\alpha:[0, a[\rightarrow[0, \infty[$ is said to belong to class $\mathcal{K}$ if it is strictly increasing and $\alpha(0)=$ 0 .

- A continuous function $\beta$ : $[0, a[\times[0, \infty[\mapsto[0, \infty[$ is said to belong to class $\mathcal{K} \mathcal{L}$ if, for each fixed $s$, the mapping $\beta(r, s)$ belongs to class $\mathcal{K}$ with respect to $r$ and for each fixed $r$, the mapping $\beta(r, s)$ is decreasing with respect to $s$ and $\beta(r, s) \rightarrow 0$ as $s \rightarrow \infty$.

Next theorem states a general result for interconnected delay and state observers.

Theorem 2: For the disturbance free system, suppose that there exist a state observer such that

$$
\dot{V}_{x} \leq-c_{x}\left\|e_{x}\right\|^{2}+c_{x \tau}\left\|e_{x}\right\|\left\|e_{\tau}\right\|
$$

and a delay observer such that

$$
\begin{aligned}
\dot{V}_{z h} \leq & -c_{z}\left\|e_{z}\right\|^{2}-c_{\tau}\left\|e_{\tau}\right\|^{2} \\
& +c_{z \tau}\left\|e_{z}\right\|\left\|e_{\tau}\right\|+c_{z x}\left\|e_{z}\right\|\left\|e_{x}\right\|
\end{aligned}
$$

with $c_{x}, c_{z}, c_{\tau}, c_{x \tau}, c_{z x}, c_{z \tau}>0$. An appropriate choice of $c_{x}, c_{z}, c_{\tau}$, guarantees the existence of a class $\mathcal{K} \mathcal{L}$ function $\beta$, a finite time $T$ and an initial time $t_{0}$ such that the error $e=$ $\left[e_{x}^{T}, e_{z}^{T}, z_{\tau}\right]^{T}$ of the disturbed system is uniformly bounded

$$
\|e(t)\| \leq \beta\left(\left\|x\left(t_{0}\right)\right\|, t-t_{0}\right), \quad \text { for all } \quad t_{0} \leq t \leq t_{0}+T
$$

and

$$
\|e(t)\| \leq \delta, \quad \text { for all } t \geq t_{0}+T .
$$

Remark 3.1: The constants $c_{x}, c_{z}, c_{\tau}, c_{x \tau}, c_{z x}, c_{z \tau}$ will depend on the observer parameters. In particular, $c_{x}, c_{z}, c_{\tau}$ will be directly proportional to the observer gains as it will be shown in the next section on particular examples.

Proof: First, the uniform asymptotic convergence of the disturbance free system is worked out. The Lyapunov candidate function for the interconnected system is the sum of the Lyapunov functions of the isolated systems:

$$
V=V_{x}+V_{z h}
$$


Consequently, from (5) and (6), the time derivative of $V$ satisfies

$\dot{V} \leq-\left[\left\|e_{x}\right\|,\left\|e_{z}\right\|\left\|e_{\tau}\right\|\right]\left[\begin{array}{ccc}c_{x} & -\frac{1}{2} c_{z x} & -\frac{c_{x \tau}}{2} \\ -\frac{1}{2} c_{z x} & c_{z} & -\frac{c_{z \tau}}{2} \\ -\frac{c_{x \tau}}{2} & -\frac{c_{z \tau}}{2} & c_{\tau}\end{array}\right]\left[\begin{array}{l}\left\|e_{x}\right\| \\ \left\|e_{z}\right\| \\ \left\|e_{\tau}\right\|\end{array}\right]$.

In order to have $\dot{V}$ negative, the matrix

$$
S=\left[\begin{array}{ccc}
c_{x} & -\frac{1}{2} c_{z x} & -\frac{c_{x \tau}}{2} \\
-\frac{1}{2} c_{z x} & c_{z} & -\frac{c_{z \tau}}{2} \\
-\frac{c_{x \tau}}{2} & -\frac{c_{z \tau}}{2} & c_{\tau}
\end{array}\right]
$$

has to be positive definite: then, the leading principal minors of $S$ must be positive i.e.

$$
\left\{\begin{array}{l}
c_{x}>0 \\
c_{x} c_{z}-\frac{1}{4} c_{z x}^{2}>0 \\
c_{x} c_{z} c_{\tau}-\frac{1}{4} c_{z \tau}^{2} c_{x}-\frac{1}{4} c_{x \tau}^{2} c_{z}-\frac{1}{4} c_{z x} c_{x \tau} c_{z \tau}-\frac{1}{4} c_{\tau} c_{z x}^{2}>0
\end{array}\right.
$$

As reminded in Remark ; the terms $c_{x}, c_{z}, c_{\tau}$ are proportional to the observers gains so they can be chosen sufficiently large in order to make $S$ definite positive.

The uniform boundedness of the disturbed system directly results from the application of Lemma 9.3 in [12].

\section{PARTICULAR SOLUTIONS FOR STATE AND DELAY OBSERVERS}

In this section, some observation solutions are exhibited to illustrate the general consideration of above section. First, a state observer is designed and it is shown that it complies with (5). Then, a delay observer is presented and condition (6) is checked.

\section{A. State Observer}

A Kalman-Like observer [4] is chosen to observe the state of system (1). This choice has been made because of its simplicity; indeed, it only has one tuning parameter. Observer equations are given below

$$
\left\{\begin{array}{l}
\dot{\hat{x}}=\Lambda \hat{x}+f(\hat{x})+g(y) u(t-\tilde{\tau})-S_{x}^{-1} C^{T} C(\hat{x}-x) \\
-\rho_{x} S_{x}-\Lambda^{T} S_{x}-S_{x} \Lambda+C^{T} C=0 \\
S_{x}(0)=S_{x}(0)^{T}>0
\end{array}\right.
$$

It is important to note that the input $u$ is affected by the delay estimation $\tilde{\tau}$ and not the real delay $\tau$. The dynamics of the state observation error is given by

$\dot{e}_{x}=\left(\Lambda-S_{x}^{-1} C^{T} C\right) e_{x}+f(\hat{x})-f(x)+g(y)[u(t-\tilde{\tau})-u(t-\tau)]$

Remark 4.1: In (9), it is clear from the continuity of $u$ that the error term $u(t-\tilde{\tau})-u(t-\tau)$ will tend to zero as soon as $\tilde{\tau}$ tends to $\tau$.

To prove that condition (5) holds for state observer (8), the following Lyapunov candidate function is defined

$$
V_{x}=e_{x}^{T} S_{x} e_{x}
$$

with $S_{x}$ the unique symmetric positive definite matrix solution of the Lyapunov equation in (8). From [4], there exist $\bar{\delta}_{x}, \underline{\delta}_{x}>0$ such that

$$
\underline{\delta}_{x} I_{n} \leq S_{x} \leq \bar{\delta}_{x} I_{n}
$$

where $I_{n}$ is the identity matrix of dimension $n$. Taking the time-derivatives of $V_{x}$ along the trajectories of (1) leads to

$$
\begin{aligned}
\dot{V}_{x}= & -\rho_{x} e_{x}^{T} S_{x} e_{x}-e_{x}^{T} C^{T} C e_{x}+2 e_{x}^{T} S_{x}[f(x)-f(\hat{x})]+ \\
& 2[u(t-\tilde{\tau})-u(t-\tau)] G(y)^{T} S_{x} e_{x} .
\end{aligned}
$$

Then, from equation (11) and Assumptions 1,2, one derives the inequality

$$
\begin{aligned}
\dot{V}_{x} \leq & -\rho_{x} \underline{\delta}_{x}\left\|e_{x}\right\|^{2}+2 c_{1} \bar{\delta}_{x}\left\|e_{x}\right\|^{2} \\
& +2 \bar{G} \bar{\delta}_{x}\left\|e_{x}\right\||u(t-\tilde{\tau})-u(t-\tau)| .
\end{aligned}
$$

From the mean value theorem, there exists $\kappa_{t} \in[\min (t-$ $\tau, t-\tilde{\tau}), \max (t-\tau, t-\tilde{\tau})]$ such that

$$
u(t-\tilde{\tau})-u(t-\tau)=(\tau-\tilde{\tau}) \dot{u}\left(\kappa_{t}\right)
$$

so from Assumption 4 one has

$$
\begin{aligned}
\dot{V}_{x} \leq & -\rho_{x} \underline{\delta}_{x}\left\|e_{x}\right\|^{2}+2 c_{1} \bar{\delta}_{x}\left\|e_{x}\right\|^{2} \\
& +2 \bar{G} U_{1} \bar{\delta}_{x}\left\|e_{x}\right\|\left\|e_{\tau}\right\| .
\end{aligned}
$$

Finally, taking $\rho_{x}$ sufficiently large, the above equation can be rewritten as follows

$$
\dot{V}_{x} \leq-c_{x}\left\|e_{x}\right\|^{2}+c_{x \tau}\left\|e_{x}\right\|\left\|e_{\tau}\right\|
$$

with $c_{x}, c_{x \tau}>0$. Consequently, this state observer satisfies (5). In the simulation section, this observer will be tested with the delay observer designed thereafter.

\section{B. New Delay Observer}

This delay observer is based on the minimization of the criterion

$$
J(\tilde{\tau})=[u(t-\tilde{\tau})-u(t-\tau)]^{2}
$$

on $\left[\tau_{\min }, \tau_{\max }\right]$. However, usually $u(t-\tau)$ is not available. The idea is to compute an estimation $\tilde{u}(t-\tau)$ of $u(t-\tau)$ and to optimize the approximated criterion

$$
\tilde{J}(\tilde{\tau})=[u(t-\tilde{\tau})-\tilde{u}(t-\tau)]^{2} .
$$

Note that the accuracy of the delay estimation is tightly related to the accuracy of $\tilde{u}(t-\tau)$. The following dynamics, inspired from [16], is proposed for the delay estimation

$$
\dot{\tilde{\tau}}=-\rho_{\tau}[u(t-\tilde{\tau})-\tilde{u}(t-\tau)] \frac{\partial u(t-\tilde{\tau})}{\partial \tilde{\tau}} .
$$

Since

$$
\frac{\partial u(t-\tilde{\tau})}{\partial \tilde{\tau}}=-\frac{\partial u(t-\tilde{\tau})}{\partial t},
$$

it results that the dynamics of $\tilde{\tau}$ is

$$
\dot{\tilde{\tau}}=\rho_{\tau}[u(t-\tilde{\tau})-\tilde{u}(t-\tau)] \dot{u}(t-\tilde{\tau}) .
$$

In practice, the time derivative of $u$ is not always available so it will be approximated by

$$
\mu(t)=\frac{u(t-\tilde{\tau})-u(t-\tilde{\tau}-h)}{h}
$$

where $h$ is an artificial delay. Thus, equation (19) becomes

$$
\dot{\tilde{\tau}}=\rho_{\tau}[u(t-\tilde{\tau})-\tilde{u}(t-\tau)] \mu(t) .
$$


In the sequel, it is assumed that there exist intervals $I$ such that $t-\tilde{\tau}(t)-h \in I, t-\tau(t) \in I$ and $|\dot{u}(s)|>\alpha$ for all $s \in I$ (Assumption 5). To estimate $u(t-\tau)$, the ESO (Extended System Observer) technique is used (see [18] for references on this method). To achieve a better accuracy [20], it has been decided to also observe $\dot{u}(t-\tau)$. Therefore, the extended system reads as

$$
\left\{\begin{array}{l}
\dot{z}=\Lambda_{z} z+f_{z}(x)+\Gamma_{z}(t) \\
\dot{\tau}(t)=\eta_{\tau}(t) \\
y=C_{z} z=[1,0,0] z=x_{n}
\end{array}\right.
$$

with $z=\left[x_{n}, u(t-\tau), \dot{u}(t-\tau)\right]^{T}$,

$$
\Lambda_{z}=\left[\begin{array}{ccc}
0 & G(y) & 0 \\
0 & 0 & 1 \\
0 & 0 & 0
\end{array}\right]
$$

$f_{z}(x)=[f(x), 0,0]^{T}$ and $\Gamma_{z}(t)=\left[0,0, \eta_{u}(t)\right]^{T}$ where $\eta_{u}$ is unknown. The output of (22) is $x_{n}$; however, $x_{n}$ is unknown but the value $\hat{x}_{n}$ given by state observer (8) will be used in the observer design. The equations of the delay observer are given below:

$$
\left\{\begin{array}{l}
\dot{\tilde{z}}=\Lambda_{z} \tilde{z}+f_{z}(\hat{x})-S_{z}^{-1} C_{z}^{T}\left[\tilde{x}_{n}-\hat{x}_{n}\right] \\
\dot{S}_{z}=-\rho_{z} S_{z}-\Lambda_{z}^{T} S_{z}-S_{z} \Lambda_{z}+C_{z}^{T} C_{z} \\
S_{z}(0)=S_{z}(0)^{T}>0
\end{array}\right.
$$

and

$$
\dot{\tilde{\tau}}=\rho_{\tau}\left[u(t-\tilde{\tau})-C_{u} \tilde{z}\right] \mu(t)
$$

with $\tilde{z}=\left[\tilde{x}_{n}, \tilde{u}(t-\tau), \tilde{\dot{u}}(t-\tau)\right]^{T}, C_{z}=[1,0,0]$ and $C_{u}=$ $[0,1,0]$. To sum up, the observer can be divided into two parts. The first part (23) that allows to observe $u(t-h)$ and the estimation delay dynamics in (24). Reminding that

$$
\begin{aligned}
\tilde{x}_{n}-\hat{x}_{n} & =C_{z} \tilde{z}-C_{z} z+C_{z} z-C_{n} \hat{x} \\
& =C_{z} e_{z}-C_{n} e_{x}
\end{aligned}
$$

with $C_{n} \in \mathbb{R}^{n}$ and $C_{n}=[0, \ldots, 0,1]$, then the dynamics of the delay observation errors are

$$
\begin{gathered}
\dot{e}_{z}=\left[\Lambda_{z}-S_{z}^{-1} C_{z}^{T} C_{z}\right] e_{z}+f_{n}(\hat{x})-f_{n}(x)+ \\
S_{z}^{-1} C_{z}^{T} C_{n} e_{x}-\Gamma_{z}(t)
\end{gathered}
$$

and

$$
\dot{e}_{\tau}=\rho_{\tau}[u(t-\tilde{\tau})-\tilde{u}(t-\tau)] \mu(t)-\eta_{\tau}(t) .
$$

Note that the dynamics $\Gamma_{z}$ and $\eta_{\tau}$ are unknown and will be considered as a perturbation of the error dynamics. To analyze the stability of this observer, the following Lyapunov candidate function is defined

$$
V_{z \tau}=V_{z}+V_{\tau}
$$

with $V_{z}=e_{z}^{T} S_{z} e_{z}$ and $V_{\tau}=\frac{1}{2}(\hat{\tau}-\tau)^{2}$. Taking the time derivative of $V_{\tau}$ along the trajectories of (27) gives

$$
\dot{V}_{\tau}=\rho_{\tau}(\tilde{\tau}-\tau)[u(t-\tilde{\tau})-\tilde{u}(t-\tau)] \mu-(\tilde{\tau}-\tau) \eta_{\tau}
$$

Since

$$
u(t-\tilde{\tau})-\tilde{u}(t-\tau)=u(t-\tilde{\tau})-u(t-\tau)-C_{u} e_{z}(t)
$$

then from the mean value theorem there exist $\phi_{t} \in[\min (t-$ $\tau, t-\tilde{\tau}), \max (t-\tau, t-\tilde{\tau})]$ such that

$$
u(t-\tilde{\tau})-\tilde{u}(t-\tau)=(\tau-\tilde{\tau}) \dot{u}\left(\phi_{t}\right)-C_{u} e_{z}(t) .
$$

In the same way, there exists $\theta_{t} \in[t-\tilde{\tau}-h, t-\tilde{\tau}]$ such that

$$
\mu(t)=\dot{u}\left(\theta_{t}\right)=\frac{u(t-\tilde{\tau})-u(t-\tilde{\tau}-h)}{h}
$$

so one gets

$\dot{V}_{\tau}=-\rho_{\tau}(\hat{\tau}-\tau)^{2} \dot{u}\left(\phi_{t}\right) \dot{u}\left(\theta_{t}\right)-(\hat{\tau}-\tau)\left[\rho_{\tau} \dot{u}\left(\theta_{t}\right) C_{u} e_{z}+\eta_{\tau}\right]$.

Provided that $t-\tilde{\tau}-h, \theta_{t}$ and $\phi_{t}$ are in interval $I$, the terms $\dot{u}\left(\phi_{t}\right)$ and $\dot{u}\left(\theta_{t}\right)$ have the same sign so it follows that

$\dot{V}_{\tau}(t) \leq-\rho_{\tau} \alpha^{2}\left\|e_{\tau}\right\|^{2}+\rho_{\tau} U_{1}\left\|e_{\tau}\right\|\left\|e_{z}\right\|+\rho_{\tau}\left\|e_{\tau}\right\| \sup _{s \leq t}\left|\eta_{\tau}(s)\right|$

with $U_{1}$ defined in Assumption 4. As a result, for $\eta_{\tau}=0$, the maximization

$$
\dot{V}_{\tau} \leq-\rho_{\tau} \alpha^{2}\left\|e_{\tau}\right\|^{2}+\rho_{\tau} U_{1}\left\|e_{z}\right\|\left\|e_{\tau}\right\|
$$

can be deduced from the above equation.

Taking the time derivative of $V_{z}$ along the trajectories of (26) leads to

$$
\begin{aligned}
\dot{V}_{z}= & -\rho_{z} e_{z}^{T} S_{z} e_{z}-e_{z}^{T} C_{z} C_{z} e_{z}+2 e_{z}^{T} S_{z}[f(\hat{x})-f(x)] \\
& +2 e_{z}^{T} C_{z}^{T} C_{n} e_{x}
\end{aligned}
$$

for $\Gamma_{z}=0$. Besides, there exists $t_{0}>0$, such that for all $t \geq t_{0}, S_{z}$ verifies

$$
\underline{\delta}_{z} I_{n} \leq S_{z}(t) \leq \bar{\delta}_{z} I_{n}
$$

for $\rho_{z}$ sufficiently large and where $\underline{\delta}_{z}$ and $\bar{\delta}_{z}$ are positive constants (see [4]). Since $f$ is globally Lipschitz (Assumption 1), one can obtain the following maximization

$$
\dot{V}_{z} \leq-\rho_{z} \underline{\delta}_{z}\left\|e_{z}\right\|^{2}+c_{z x}\left\|e_{z}\right\|\left\|e_{x}\right\|
$$

with $c_{x z}>0$. Then, from (35) and (38), one gets

$\dot{V}_{z \tau} \leq-c_{z}\left\|e_{z}\right\|^{2}+c_{z x}\left\|e_{z}\right\|\left\|e_{x}\right\|-c_{\tau}\left\|e_{\tau}\right\|^{2}+c_{z \tau}\left\|e_{z}\right\|\left\|e_{\tau}\right\|$

with $c_{z}=\rho_{z} \underline{\delta}_{z}, c_{\tau}=\rho_{\tau} \alpha^{2}$ and $c_{z \tau}=\rho_{\tau} U_{1}$. Therefore, delay observer (23)-(24) satisfies condition (6).

In the next section, this new delay observation method is going to be illustrated and its performances will be compared with the results from [14].

\section{Simulation}

\section{A. A nonlinear example: the pendulum}

Simulations are carried out on a pendulum whose equations are

$$
\left\{\begin{array}{l}
\dot{x}(t)=\left[\begin{array}{c}
x_{2} \\
-\frac{g}{l} \sin x_{1}-\frac{k}{m_{p}} x_{2}
\end{array}\right]+\left[\begin{array}{c}
0 \\
\frac{1}{m_{p} l^{2}}
\end{array}\right] u(t-\tau) \\
y(t)=C x(t)=x_{1}(t),
\end{array}\right.
$$

with $g=9.81 \mathrm{~m} \cdot \mathrm{s}^{-2}$ the acceleration due to gravity, $m_{p}=0.2 \mathrm{~kg}$ the mass of the bob, $l=0.5 \mathrm{~m}$ the length of the rod and $k=0.3 \mathrm{~kg} . \mathrm{s}^{-1}$ the friction coefficient. 
Details of the modeling are given in the Example section of [12]. The identification with (1) leads to $\Lambda=\left[\begin{array}{ll}0 & 1 \\ 0 & 0\end{array}\right]$, $f(x)=\left[0,-\frac{g}{l} \sin x_{1}-\frac{k}{m_{p}} x_{2}\right]^{T}$ and $G(y)=\frac{1}{m_{p} l^{2}}$. The functions $f$ and $G$ satisfy Assumptions 1 and 2 . In the simulation, the observation of a piecewise constant delay and a sine delay are implemented; the exact expression of the delay is given below:

$$
\tau(t)= \begin{cases}0.4 & \text { for } 0 \leq t<20 \\ 1 & \text { for } 20 \leq t<40 \\ 0.6 & \text { for } 40 \leq t<60 \\ 0.6+0.45 \sin (0.2 t) & \text { for } t \geq 60\end{cases}
$$

Note that $\tau$ verifies Assumption 3 with $\tau_{\min }=0.1 \mathrm{~s}$ and $\tau_{\max }=1.2 \mathrm{~s}$. To analyze the influence of the input dynamics on the delay estimation, two signals are used:

- $u_{1}(t)=0.1 t$

- $u_{2}(t)=\sin (0.2 t)$.

Both signals satisfy Assumption 4. However, only $u_{1}$ satisfies the delay observability condition of Theorem 1 . For $u_{2}$, the observer will go through observation singularities when $\dot{u}_{2}=$ 0 . The state observer

$$
\left\{\begin{aligned}
\dot{\hat{x}}(t)= & \Lambda \hat{x}(t)+\left[\begin{array}{c}
0 \\
-\frac{g}{l} \sin \hat{x}_{1}-\frac{k}{m_{p}} \hat{x}_{2}
\end{array}\right]+\left[\begin{array}{c}
0 \\
\frac{1}{m_{p} l^{2}}
\end{array}\right] u(t) \\
& -S^{-1} C^{T}\left(\hat{x}_{1}-x_{1}\right) \\
\dot{S}= & -\rho S-\Lambda^{T} S-S \Lambda+C^{T} C
\end{aligned}\right.
$$

and the delay observer

$$
\left\{\begin{array}{l}
\dot{\hat{z}}=\Lambda_{z} \hat{z}+f_{z}(\hat{x})-S_{z}^{-1} C_{z}^{T}\left[C_{z} \hat{z}-C_{2} \hat{x}\right] \\
\dot{S}_{z}=-\rho_{z} S_{z}-\Lambda_{z}^{T} S_{z}-S_{z} \Lambda_{z}+C_{z}^{T} C_{z} \\
S_{z}(0)=S_{z}(0)^{T}>0 \\
\dot{\tilde{\tau}}(t)=\rho_{\tau}\left[u(t-\tilde{\tau}(t))-C_{u} \hat{z}\right] \mu(t) \\
\mu(t)=\frac{u(t-\tilde{\tau})-u(t-\tilde{\tau}-h)}{h}
\end{array}\right.
$$

with $\wedge_{z}=\left[\begin{array}{ccc}0 & \frac{1}{m_{p} l^{2}} & 0 \\ 0 & 0 & 1 \\ 0 & 0 & 0\end{array}\right], f_{z}(\hat{x})=\left[-\frac{g}{l} \sin \hat{x}_{1}-\right.$ $\left.\frac{k}{m_{p}} \hat{x}_{2}, 0,0\right]^{T} C_{z}=[1,0,0], C_{2}=[0,1], C_{u}=[0,1,0]$, $h=0.1$ are tested. On Figure 2, the ramp input $u_{1}$ is applied to system (40). Since $\ddot{u}_{1}=0$, then $\tilde{u}(t-\tau) \rightarrow u(t-\tau)$. When the delay is constant, $\eta_{t}=0$ so the delay estimate converges asymptotically to the real value of the delay. Consequently, the state estimation error asymptotically converges to zero. When the delay is time-varying, an unavoidable error due to the delay dynamics appears. As a consequence, the state observation error can converge asymptotically to zero anymore: it converges in a ball around the origin (less than $1 \%$ error). On Figure 3, the sine input $u_{2}$ is applied to system (40). In this case $\ddot{u}_{2}$ is different from zero so $\tilde{u}(t-\tau)$ tends to a neighborhood of $u(t-\tau)$. Consequently, this small error on $\tilde{u}(t-\tau)$ causes a small error on $\tilde{\tau}$ and finally on $\hat{x}$. However, the radius of the errors $\left\|e_{x}\right\|$ is small (about $1 \%$ error) and the delay estimation is quite accurate.

Remark 5.1: The input $u_{2}$ presents observation singularities because its time derivative cancels in some isolated
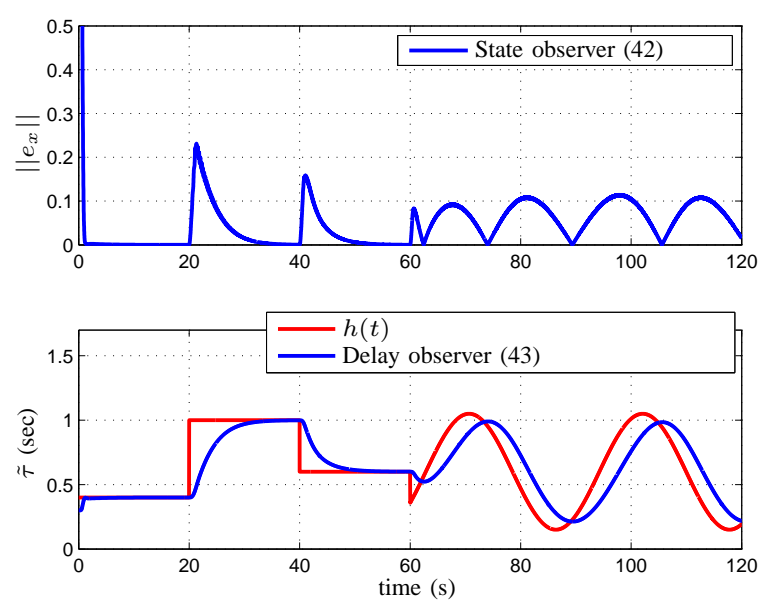

Fig. 2. $u(t)=u_{1}(t)(\mathrm{ramp}), \rho_{x}=10, \rho_{z}=10, \rho_{\tau}=40$
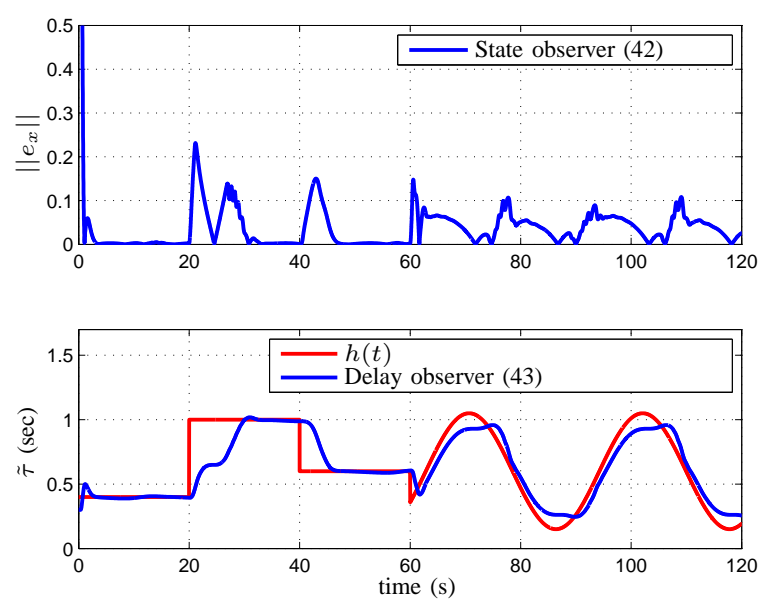

Fig. 3. $u(t)=u_{2}(t)$ (sine), $\rho_{x}=10, \rho_{z}=10, \rho_{\tau}=40$

points. However, this delay observer is not sensitive to the observability loss (persistence property).

These errors can be attenuated by increasing the observers gains $\rho_{x}, \rho_{z}$ and $\rho_{\tau}$. On Figure 4 , the same input $u_{2}$ is applied but the gains have been increased. It is clear that the estimation errors are reduced. However, the gains amplitude has to been chosen as a trade off between, noise amplification and observation accuracy in practice. Theoretical results developed in preceding sections are confirmed by simulations and an interesting property of the new delay observation technique is highlighted: the non sensitivity to observation singularity in $\dot{u}=0$. In the next subsection, a comparison with the results of [14] is presented.

\section{B. A comparison with delay state observer in [14]}

The linear second order system of [14] is reminded:

$$
\left\{\begin{array}{l}
\dot{x}(t)=\left[\begin{array}{cc}
0 & 1 \\
-2 & -3
\end{array}\right] x(t)+\left[\begin{array}{l}
0 \\
1
\end{array}\right] u(t-\tau) \\
y(t)=C x(t)=x_{1}(t) .
\end{array}\right.
$$

The system is observable and Assumptions 1 and 2 are verified since the system is linear. The same delay $\tau$ and input $u_{2}$, as defined in subsection V-A, are used. 

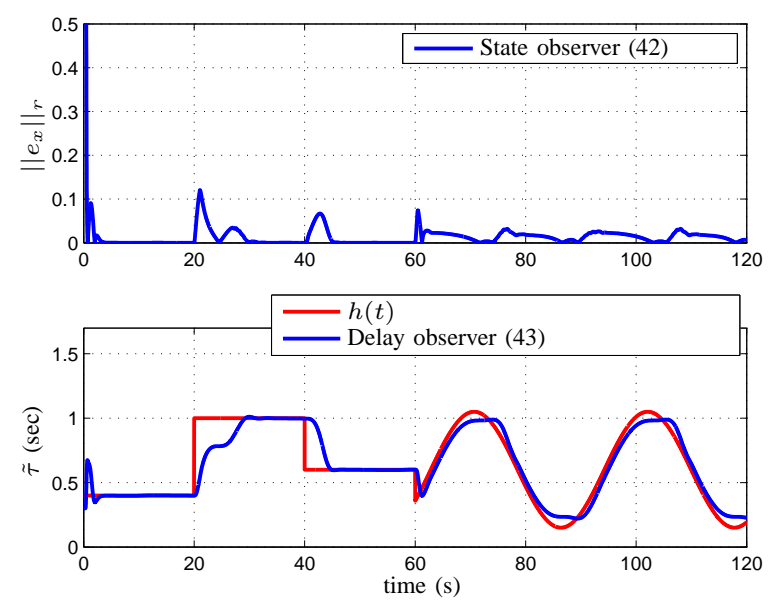

Fig. 4. $u(t)=u_{2}(t)$ (sine), $\rho_{x}=20, \rho_{z}=20, \rho_{\tau}=50$

Figure 5 shows the results of the interconnected observers scheme developed in the present paper and the method of [14]. The observation errors on the state is better for our method of interconnected observers because it does not have large transients. Similarly, delay observer (43) performs better than the one of [14] since it is less sensitive to the observation singularity $\left(\dot{u}_{2}=0\right)$. This illustrates the improvement of the presented method over the result in [14].

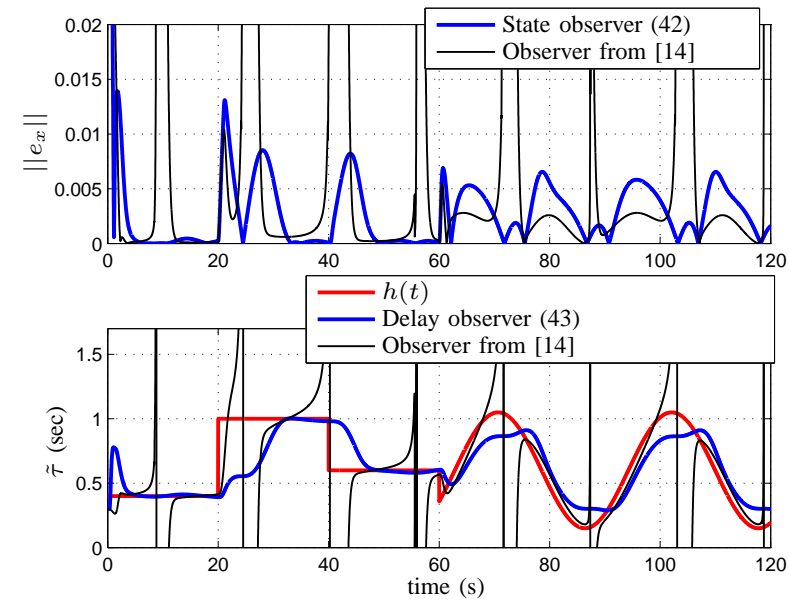

Fig. 5. Comparison between the observer in [14] with $u(t)=u_{2}(t)$ (sine)

\section{CONCLUSION}

In this paper, a new scheme of interconnected observers is proposed to estimate both the state and the delay of nonlinear systems with input delay. The advantage of decoupling state and delay observation is to allow an independent tuning of both observers and to use standard state observers. A new delay estimation technique based on an optimization method has been presented and its efficiency has been illustrated through simulations. Future developments aim at including a controller that will compute a prediction using delay and state estimations from an interconnected observer. The design of an adaptive gain for the delay observer is also taken into consideration for further study.

\section{REFERENCES}

[1] Z. Artstein, "Linear systems with delayed controls: A reduction," IEEE Trans. Autom. Control, vol. 27, no. 4, pp. 869-879, 1982.

[2] L. Belkoura, J.-P. Richard, and M. Fliess, "Parameters estimation of systems with delayed and structured entries," Automatica, vol. 45, no. 5, pp. 1117-1125, 2009.

[3] G. Besançon, "An overview on observer tools for nonlinear systems," in Nonlinear Observers and Applications, ser. Lecture Notes in Control and Information Sciences, G. Besançon, Ed. Springer, 2007, vol. 363, pp. $1-31$.

[4] G. Besançon, G. Bornard, and H. Hammouri, "Observer synthesis for a class of nonlinear control systems," European Journal of Control, vol. 2, no. 3, pp. 176-192, 1996.

[5] D. Bresch-Pietri, J. Chauvin, and N. Petit, "Adaptive control scheme for uncertain time-delay systems," Automatica, vol. 48, no. 8, pp. 1536-1552, 2012.

[6] S. Diop, I. Kolmanovsky, P. E. Moraal, and M. van Nieuwstadt, "Preserving stability-performance when facing an unknown timedelay," Control Engineering Practice, vol. 9, no. 12, pp. 1319-1325, 2001.

[7] D. L. Gaudette and D. E. Miller, "Stabilizing a SISO LTI plant with gain and delay margins as large as desired," IEEE Trans. Autom. Control, vol. 59, no. 9, pp. 2324-2339, 2014.

[8] J.-P. Gauthier and G. Bornard, "Observability for any $u(t)$ of a class of nonlinear systems," IEEE Trans. Autom. Control, vol. 26, no. 4, pp. 922-926, 1981.

[9] A. Germani, C. Manes, and P. Pepe, "A new approach to state observation of nonlinear systems with delayed output," IEEE Trans. Autom. Control, vol. 47, no. 1, pp. 96-101, 2002.

[10] R. E. Kalman and R. S. Bucy, "New results in linear filtering and prediction theory," Journal of Fluids Engineering, vol. 83, no. 1, pp. 95-108, 1961.

[11] N. Kazantzis and R. A. Wright, "Nonlinear observer design in the presence of delayed output measurements," Systems \& Control Letters, vol. 54, no. 9, pp. 877-886, 2005.

[12] H. K. Khalil, Nonlinear Systems, 3rd ed. Prentice Hall, 2002.

[13] M. Krstic, Delay compensation for nonlinear, adaptive, and PDE systems, ser. Systems and Control: Fundations and Applications. Springer, 2009.

[14] V. Léchappé, J. de León Morales, E. Moulay, F. Plestan, and A. Glumineau, "Delay and state observer for SISO LTI systems," in American Control Conference, Chicago, USA, 2015.

[15] V. Léchappé, E. Moulay, F. Plestan, A. Glumineau, and A. Chriette, "New predictive scheme for the control of LTI systems with input delay and unknown disturbances," Automatica, vol. 52, no. 2, pp. 179$184,2015$.

[16] D. Liberzon and R. Tempo, "Common Lyapunov functions and gradient algorithms," IEEE Trans. Autom. Control, vol. 49, no. 6, pp. 990-994, 2004.

[17] D. G. Luenberger, "Observing the state of a linear system," IEEE Transactions on Military Electronics, vol. 8, no. 2, pp. 74-80, 1964.

[18] R. Madonski and P. Herman, "Survey on methods of increasing the efficiency of extended state disturbance observers," ISA Transactions, vol. 56, no. 5, pp. 18-27, 2015.

[19] A. Polyakov, D. Efimov, W. Perruquetti, and J.-P. Richard, "Output stabilization of time-varying input delay systems using interval observation technique," Automatica, vol. 49, no. 11, pp. 3402-3410, 2013.

[20] A. Radke and G. Zhiqiang, "A survey of state and disturbance observers for practitioners," in American Control Conference, Minneapolis, USA, 2006.

[21] A. Seuret, T. Floquet, J.-P. Richard, and S. K. Spurgeon, "Observer design for systems with non small and unknown time-varying delay," in Topics in Time Delay Systems, ser. Lecture Notes in Control and Information Sciences. Springer Berlin Heidelberg, 2009, vol. 388, pp. 233-242.

[22] S. Torkamani and E. A. Butcher, "Delay, state, and parameter estimation in chaotic and hyperchaotic delayed systems with uncertainty and time-varying delay," International Journal of Dynamics and Control, vol. 1, no. 2, pp. 135-163, 2013.

[23] J. Tuch, A. Feuer, and Z. J. Palmor, "Time delay estimation in continuous linear time-invariant systems," IEEE Trans. Autom. Control, vol. 39, no. 4, pp. 823-827, 1994.

[24] K. Watanabe and M. Ito, "An observer for linear feedback control laws of multivariable systems with multiple delays in controls and outputs," Systems \& Control Letters, vol. 1, no. 1, pp. 54-59, 1981. 